\title{
Transcriptome analysis reveals the mechanism by which spraying diethyl aminoethyl hexanoate after anthesis regulates wheat grain filling
}

\author{
Daxing Wen ${ }^{\dagger}$, Yan Li ${ }^{\dagger}$, Lifeng He and Chunqing Zhang*
}

\begin{abstract}
Background: Diethyl aminoethyl hexanoate (DA-6), a plant growth regulator, has many beneficial effects on agricultural production. DA-6 has been applied to many plant species, but the molecular mechanism by which spraying DA-6 after anthesis regulates wheat grain filling is still unknown.

Results: In this study, we used four DA-6 concentrations: C0 (0 g/L), C2 (2 g/L), C4 (4 g/L), and C6 (6 g/L). The results showed that C4 and C6 led to a significantly higher 1000-grain weight and seed protein content than C0 during two wheat growing seasons. We then subjected samples at 24 days after anthesis (at which point the grain weight increased rapidly) to transcriptome analysis. Flag leaf (L), seed (S), and stem (T) samples under C6 and C0 were used for RNA-seq. The seed samples under C6 compared with C0 (S6vsSO) presented the most differentially expressed genes (DEGs; 2164). Plant hormone signal transduction $\left(p=1.97 \times 10^{-4}\right)$, protein processing in the endoplasmic reticulum ( $\left.E R ; p=9.04 \times 10^{-11}\right)$ and starch and sucrose metabolism $\left(p=1.90 \times 10^{-10}\right)$ pathways were the most markedly enriched pathways in the flag leaves, stems, and seeds, respectively. DEGs involved in sucrose synthesis in the flag leaves, protein processing in ER in the stems, and starch synthesis and protein processing in ER in the seeds were significantly upregulated under C6 compared with CO.

Conclusions: Overall, we propose a model for spraying DA-6 after anthesis to regulate metabolic pathways in wheat, which provides new insights into wheat in response to DA-6.
\end{abstract}

Keywords: Diethyl aminoethyl hexanoate, Transcriptome analysis, Grain weight, Seed protein content, Wheat

\section{Background}

Diethyl aminoethyl hexanoate (DA-6), an artificial tertiary amine, is a plant growth regulator that has been applied to many plant species, such as maize, cotton, soybean, peanut, and tomato [1-3]. Extensive studies have demonstrated that DA- 6 has many beneficial effects on agricultural production, including increasing the photosynthetic rate and yields, promoting biomass accumulation, and improving germination and seedling establishment of naturally and artificially aged soybean seeds [2, 4]. Moreover, DA-6 can accelerate microalgal

\footnotetext{
* Correspondence: cqzhang@sdau.edu.cn; cqzhangsdau@163.com ${ }^{\dagger}$ Daxing Wen and Yan Li contributed equally to this work.

State Key Laboratory of Crop Biology, Agronomy College, Shandong Agricultural University, Tai'an, Shandong Province 271018, People's Republic of China
}

(c) The Author(s). 2019 Open Access This article is distributed under the terms of the Creative Commons Attribution 4.0 International License (http://creativecommons.org/licenses/by/4.0/), which permits unrestricted use, distribution, and reproduction in any medium, provided you give appropriate credit to the original author(s) and the source, provide a link to the Creative Commons license, and indicate if changes were made. The Creative Commons Public Domain Dedication waiver (http://creativecommons.org/publicdomain/zero/1.0/) applies to the data made available in this article, unless otherwise stated. biodiesel production [5]. Combining DA-6 and high light can promote astaxanthin accumulation in green microalgae [6]. DA-6 also plays important roles in improving the defence response of plants under diverse environmental stresses, such as salinity stress, chilling stress and heavy metal stress [7-9]. DA-6 can be used to alleviate salinity stress by inducing the advantageous effects of salinity tolerance and decreasing oxidative damage [10]. Foliar sprays of DA-6 can increase cadmium extraction efficiency and can alleviate metal toxicity [11]. DA-6 can also be used in combination with other plant growth regulators and/or phytohormones $[9,12,13]$. Numerous studies have shown the physiological effects of DA-6, but the molecular mechanism remains unknown. 
DA-6 has been applied at many stages, including germination, seedling growth, and flowering $[1,2]$. The dissipation half-lives of DA-6 are 1.1-2.2 days, 5.4-8.2 days and 1.5-1.9 days in pakchoi, in cotton, and in the soil, respectively [1]. Rapid degradation of DA-6 would be beneficial for the safe use of DA-6 [14]. Spraying DA-6 at the seedling stage can promote plant growth by enhancing photosynthesis and regulating hormone balance in maize and soybean [3]. However, the molecular mechanism by which spraying DA-6 after anthesis influences wheat is still unknown.

Grain weight is a crucial component of grain yield and is significantly influenced by DA-6 levels [2]. Some genes and signalling pathways that determine seed size (grain weight) have been identified, such as the ubiquitin-proteasome pathway, the mitogen-activated protein kinase signalling pathway, genes involved in G-protein signalling and phytohormones and genes that encode transcriptional regulatory factors [15]. DA-6 can increase protein contents in plants [1]. Moreover, genes, environmental conditions, and cultivation patterns also affect seed protein content, which is related to both wheat end use and seed vigour [16-19]. However, the effects of spraying DA-6 after anthesis on wheat grain weight and seed protein content remain unknown.

In this study, the results showed that spraying DA-6 after anthesis increased wheat grain weight and seed protein content. Transcriptome analysis subsequently showed that DA- 6 affected plant hormone signal transduction and sucrose synthesis in the flag leaves, protein processing in endoplasmic reticulum (ER) in the stems, and starch synthesis and protein processing in ER in the seeds. Upregulated genes involved in sucrose synthesis in the flag leaves and starch synthesis in the seeds might be associated with increased 1000-grain weight and genes involved in protein processing in ER in both the stems and seeds might contribute to enhanced seed protein content.

\section{Results}

\section{DA-6 increases wheat grain weight and seed protein} content

To investigate the effects of spraying DA-6 after anthesis on wheat grain filling, we tested four DA- 6 concentrations: C0 $(0 \mathrm{~g} / \mathrm{L}), \mathrm{C} 2(2 \mathrm{~g} / \mathrm{L}), \mathrm{C} 4(4 \mathrm{~g} / \mathrm{L})$, and C6 $(6 \mathrm{~g} / \mathrm{L})$. The C4 and C6 treatments led to significantly higher 1000-grain weight and seed protein content than did $\mathrm{C} 0$ in 2017 and 2018, respectively (Table 1). C6 showed the highest 1000grain weight during the two wheat growing seasons, which was approximately $10 \%$ higher than that of $\mathrm{C} 0$. In terms of seed protein content, $\mathrm{C} 6$ and $\mathrm{C} 4$ displayed the highest seed protein content in 2017 and 2018, respectively. These results suggested that spraying DA- 6 could increase grain weight and seed protein content in wheat.
Table 1 Effects of spraying DA-6 on the 1000-grain weight and seed protein content in 2017 and 2018

\begin{tabular}{llllll}
\hline Treatment & \multicolumn{2}{l}{ 1000-grain weight $(\mathrm{g})$} & & \multicolumn{2}{c}{ Seed protein content (\%) } \\
\cline { 2 - 3 } \cline { 6 - 6 } & 2017 & 2018 & & 2017 & 2018 \\
\hline C0 & $42.71 \mathrm{c}$ & $46.56 \mathrm{c}$ & & $12.16 \mathrm{~b}$ & $12.92 \mathrm{~b}$ \\
C2 & $45.16 \mathrm{~b}$ & $45.96 \mathrm{C}$ & & $12.07 \mathrm{~b}$ & $13.25 \mathrm{a}$ \\
$\mathrm{C} 4$ & $47.52 \mathrm{a}$ & $48.90 \mathrm{~b}$ & & $13.40 \mathrm{a}$ & $13.44 \mathrm{a}$ \\
$\mathrm{C} 6$ & $48.38 \mathrm{a}$ & $49.82 \mathrm{a}$ & & $13.64 \mathrm{a}$ & $13.34 \mathrm{a}$ \\
\hline
\end{tabular}

Transcriptome analysis of wheat grain filling in response to DA-6 levels

At the grain-filling stage, C6 showed a higher 1000-grain weight than did $\mathrm{C} 0$ from 22 to 46 days after anthesis (Fig. 1a). To explore the molecular mechanism by which spraying DA-6 after anthesis regulates wheat grain filling, we subjected samples at 24 days after anthesis (at which point the grain weight increased rapidly, Fig. 1a) to transcriptome analysis. Flag leaf (L), seed (S), and stem (T) samples under $\mathrm{C} 6$ and $\mathrm{C} 0$ were used for RNA-seq, and three biological replicates were included for each treatment (Fig. 1b). After removing adapters and sequences with low-quality regions, there remained approximately 36.57-63.60 million clean reads (Additional file 4: Table S1). Approximately $32.49-53.49$ million clean reads were then mapped to the wheat genome. These clean reads comprised 4.93-8.46\% multiple mapped reads (those mapped to multiple locations) and $70.03-83.89 \%$ uniquely mapped reads (those mapped to a single location). Subsequently, we used the DESeq R package (1.18.0) to identify differentially expressed genes (DEGs) using a false discovery rate of $<0.05$ as the cutoff. The results showed that 860 DEGs were detected in the flag leaf samples under C6 compared with C0 (L6vsL0), 2164 DEGs were detected in the seed samples under C6 compared with $\mathrm{C0}$ (S6vsS0), and 605 DEGs were detected in the stem samples under C6 compared with C0 (T6vsT0) (Fig. 1c).

To facilitate the global analysis of gene expression, these DEGs were subjected to Gene Ontology (GO) enrichment analysis. In the flag leaf samples (L6vsL0), the most significantly enriched $\mathrm{GO}$ terms were protein phosphorylation (GO: 0006468, $p=1.49 \times 10^{-14}$ ) in the biological process group, anchored component of the plasma membrane (GO: 0046658, $p=1.03 \times 10^{-3}$ ) in the cellular component group, and protein kinase activity (GO: 0004672, $p=1.28 \times$ $10^{-13}$ ) in the molecular function group (Additional file 1: Figure S1). These results indicated that spraying DA-6 after anthesis might regulate protein phosphorylation in flag leaves. Sulfate transport (GO: 0008272, $p=$ $\left.6.40 \times 10^{-6}\right)$ in the biological process group, spindle microtubule (GO: 0005876, $p=4.64 \times 10^{-2}$ ) in the cellular component, and both extracellular matrix binding (GO: 0050840, $p=6.40 \times 10^{-6}$ ) and sulfate transmembrane transporter activity (GO: 0015116, 




Fig. 1 Effects of spraying DA-6 on grain weight accumulation in 2017. We used four DA-6 concentrations: C0 (0 g/L), C2 (2 g/L), C4 (4 g/L), and C6 ( $6 \mathrm{~g} / \mathrm{L})$. a 1000-grain weight accumulation; $\mathbf{b}$ samples at 24 days after anthesis (DAA; at this point, grain weight increased rapidly) for transcriptome analysis. Flag leaf $(\mathrm{L})$, seed $(\mathrm{S})$, and stem $(\mathrm{T})$ samples under C6 and C0 were used for RNA-seq, and three biological replicates were included for each treatment. c Venn diagram of differentially expressed genes (DEGs). L6vsL0: flag leaf samples under C6 compared with C0; S6vsSO: seed samples under C6 compared with C0; T6vsTO: stem samples under C6 compared with CO

$p=6.40 \times 10^{-6}$ ) in the molecular function group represented the most markedly enriched GO terms in T6vsT0 (Additional file 2: Figure S2). Therefore, sulfate transport and sulfate transmembrane transporter activity in the stems might be involved in the response to DA-6. In S6vsS0, the most prominently enriched GO terms were carbohydrate metabolic process (GO: 0005975, $p=5.45 \times 10^{-18}$ ) in the biological process group, transcription factor TFIID complex (GO: 0005669, $\left.p=1.77 \times 10^{-16}\right)$ in the cellular component group, and hydrolase activity, hydrolyzing O-glycosyl compounds (GO: 0004553, $p=3.27 \times 10^{-19}$ ) in the molecular function group (Fig. 2). The results suggested that DA-6 affected carbohydrate metabolism and hydrolase activity in the seeds, which might be related to 1000-grain weight.

\section{ABA signal transduction and sucrose synthesis in flag} leaves are involved in the response to DA- 6 levels

To identify the metabolic pathways involved in response to DA-6 levels, we further analysed the Kyoto Encyclopedia of Genes and Genomes (KEGG) enrichment pathways. Many metabolic pathways were enriched in the flag leaves, stems, and seeds (Fig. 3). Plant hormone signal transduction was the most significantly enriched pathway in L6vsL0, followed by alpha-linolenic acid metabolism, nitrogen metabolism, starch and sucrose metabolism, and flavonoid biosynthesis. ABA 


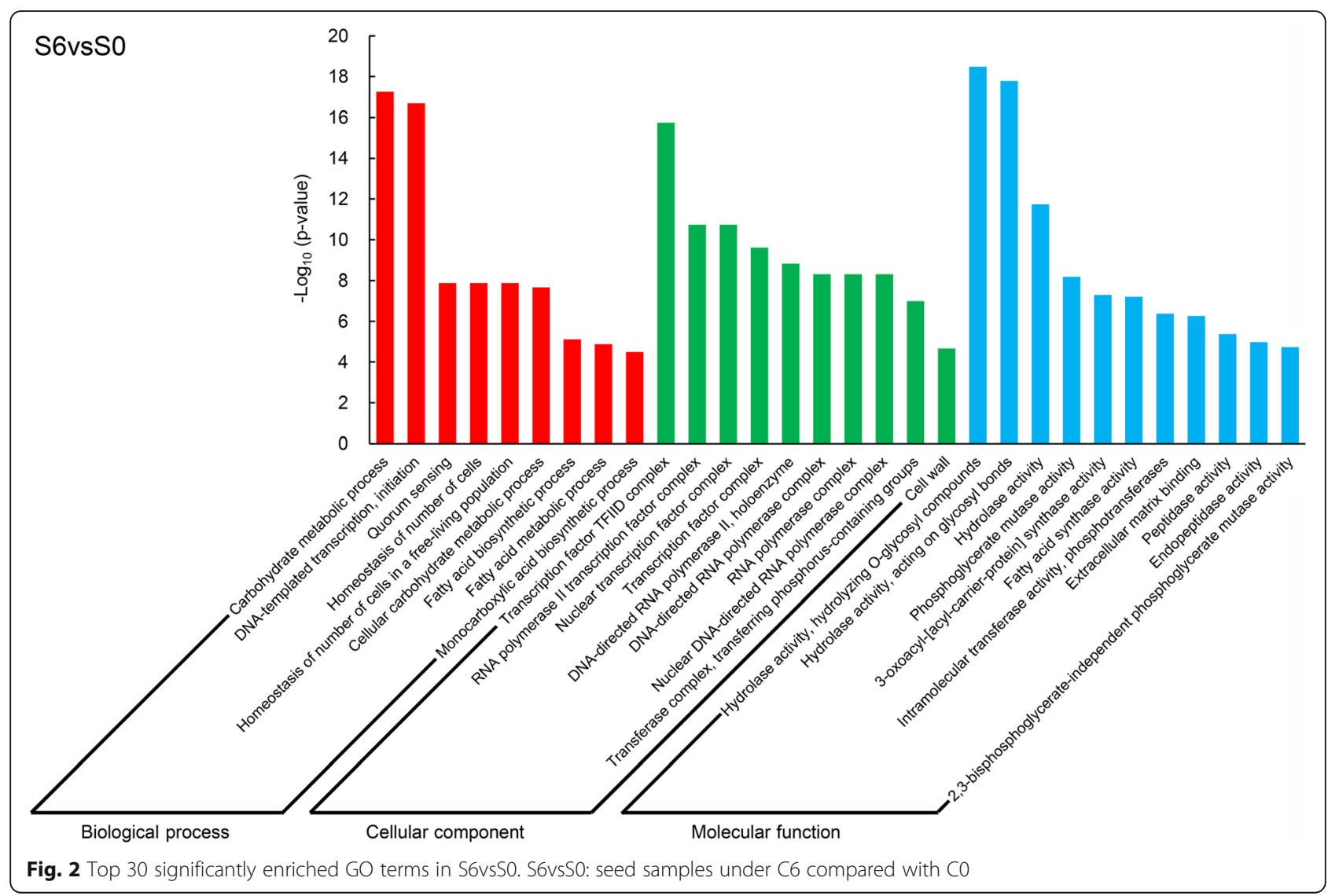

signal transduction represented the largest group within plant hormone signal transduction. Abscisic acid receptor PYR/PYL family genes (TraesCS4A02G114400, TraesCS4B02G189800 and TraesCS2D02G087500) were dramatically downregulated, while protein phosphatase 2C, serine/threonine-protein kinase SRK2 and ABA-responsive element binding factor-related genes were notably upregulated in L6vsL0 (Fig. 4, Additional file 5:
Table S2). Moreover, three DEGs (TraesCS4B02G167500, TraesCS3A02G015500 and TraesCSU02G044500) involved in sucrose synthesis and two DEGs (TraesCS5A02G513200 and TraesCS4B02G344300) related to starch decomposition were upregulated in the starch and sucrose metabolism pathway (Fig. 4, Additional file 5: Table S2). Sucrose synthase- and sucrose-phosphate synthase-related genes were upregulated in L6vsL0. The results suggested that

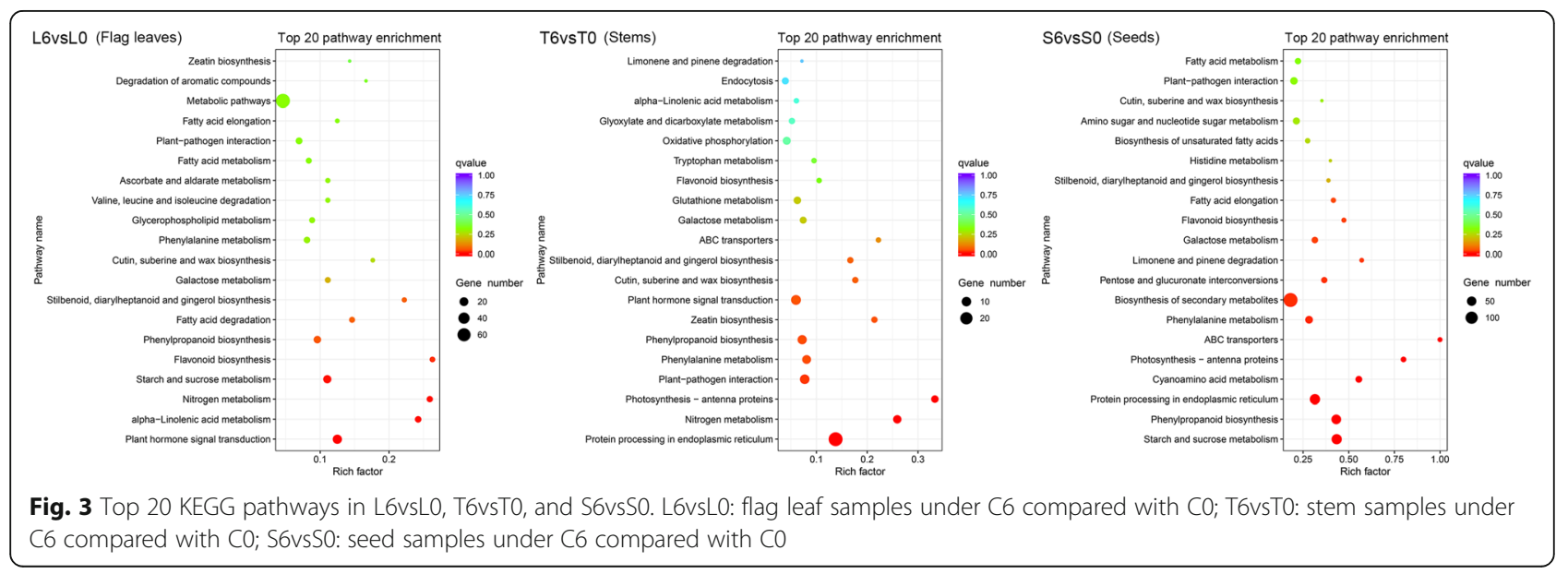




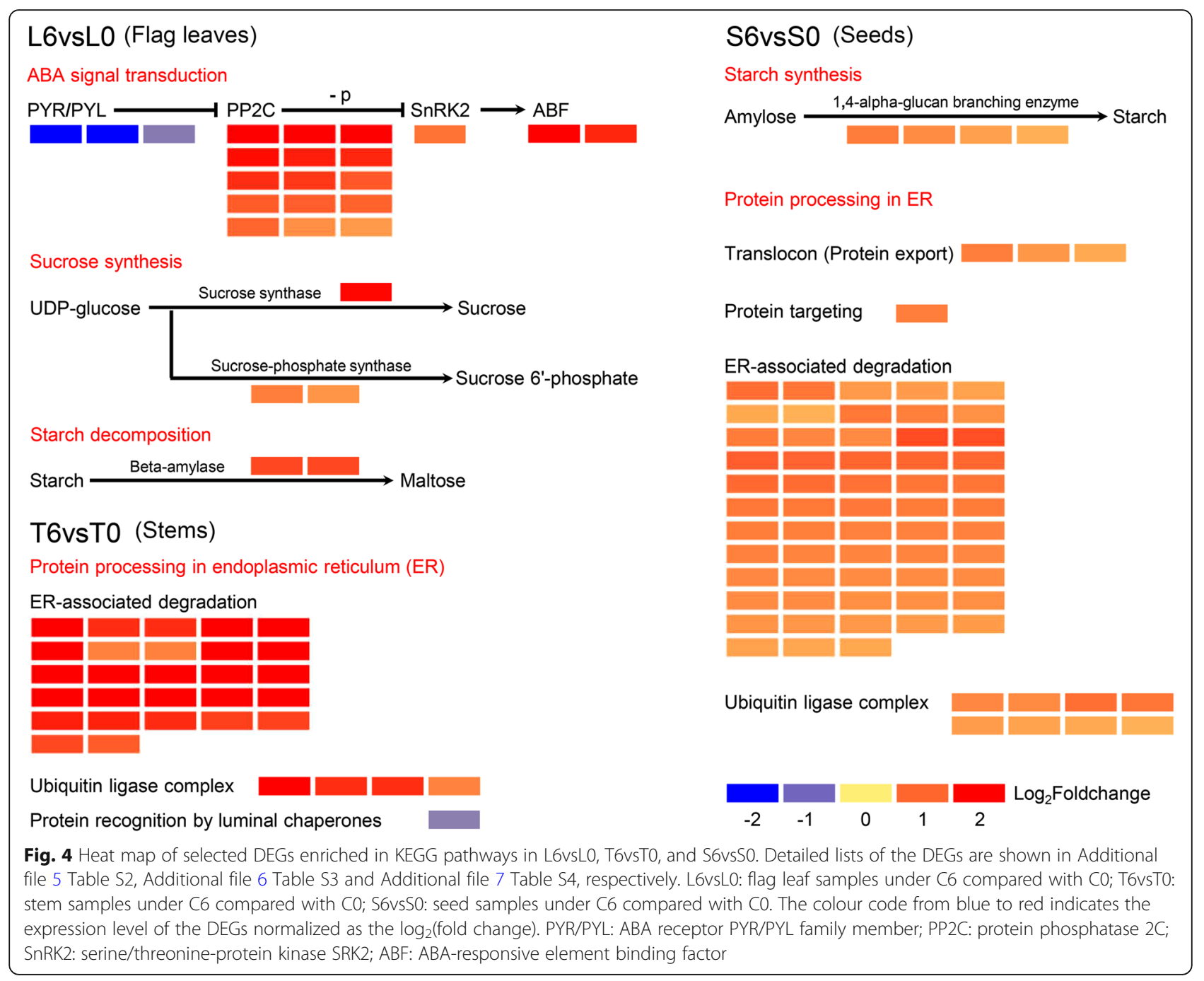

DA-6 might affect ABA signal transduction, sucrose synthesis and starch decomposition in flag leaves at the grainfilling stage.

\section{Protein processing in ER in the stems are involved in the response to DA-6 levels}

In T6vsT0, the most significantly enriched pathway was protein processing in ER, followed by nitrogen metabolism, photosynthesis - antenna proteins, plant-pathogen interactions, phenylalanine metabolism and phenylpropanoid biosynthesis (Fig. 3). Except for one downregulated gene related to protein recognition by luminal chaperones, genes involved in ER-associated degradation and the ubiquitin ligase complex were markedly upregulated in protein processing in ER (Fig. 4, Additional file 6: Table S3). Many genes involving the heat shock $70 \mathrm{kDa}$ protein, molecular chaperone HtpG, DnaJ homolog subfamily A member 2 and HSP20 family protein were upregulated in ER-associated degradation (Additional file 6: Table S3). In terms of the ubiquitin ligase complex, there were four upregulated genes. Only one downregulated gene was related to protein recognition by luminal chaperones. These results indicated that DA-6 might have influenced protein processing in ER in the stem.

\section{Starch synthesis and protein processing in ER in the seeds are involved in the response to DA-6 levels} Compared with samples from the flag leaves and stems, the seed samples showed more significantly enriched pathways. Starch and sucrose metabolism was the most significantly enriched pathway in S6vsS0, followed by phenylpropanoid biosynthesis, protein processing in ER, cyanoamino acid metabolism, photosynthesis - antenna proteins, ABC transporters, phenylalanine metabolism, biosynthesis of secondary metabolites, pentose and glucuronate interconversion, limonene and pinene degradation, galactose metabolism, flavonoid biosynthesis and fatty acid elongation (Fig. 3). In starch and sucrose metabolism, four 1,4-alpha-glucan branching enzyme 
genes (TraesCS7A02G549100, TraesCS7D02G535400, TraesCS7D02G535500 and TraesCS7B02G472500), which are involved in starch synthesis, were upregulated (Fig. 4, Additional file 7: Table S4). This phenomenon might be one of the reasons that the C6 treatment showed a higher 1000-grain weight than did C0. S6vsS0 had more genes enriched in protein processing in ER than did T6vsT0. All genes enriched in protein processing in ER in S6vsS0 were upregulated. In addition to the genes involved in ERassociated degradation and the ubiquitin ligase complex, translocon (protein export)- and protein targeting-related genes also were upregulated in S6vsS0. Many genes involving the heat shock $70 \mathrm{kDa}$ protein, molecular chaperone HtpG, HSP20 family proteins and ubiquitin conjugation factor E4 were upregulated in ER-associated degradation in S6vsSo (Additional file 7: Table S4). The number of genes enriched in the ubiquitin ligase complex in S6vsS0 was twice that in T6vsT0. These results suggested that protein processing in ER in the seeds was involved in the response to DA-6 levels, which might be related to seed protein content.

\section{Validation of RNA-Seq data by qRT-PCR}

To validate the RNA-seq results, we randomly selected six DEGs for qRT-PCR assays. Among the DEGs, two were upregulated in L6vsL0, S6vsS0 and T6vsT0 (Fig. 5). All six DEGs in the qRT-PCR assays were essentially consistent with their transcript abundance changes identified by RNA-seq, suggesting the transcriptome data were reliable. Although the fold change of TraesCS4B02G211700 according to qRT-PCR was lower than that in RNA-seq data, TraesCS4B02G211700 was upregulated in both the qRTPCR and RNA-seq data.

\section{Discussion}

Extensive studies have shown that DA- 6 can be applied at different stages and can affect various aspects of plants [1-3]. However, the mechanism of wheat at the grain-filling stage in response to DA-6 is still unknown. In this study, spraying DA- 6 one week after anthesis was used to study the effects of DA- 6 on grain filling. DA-6 treatments at the $\mathrm{V}_{3}$ stage affect many aspects of corn and soybean seedlings, such as increasing plant height and leaf area, improving the root-to-shoot ratio, and enhancing photosynthesis [3]. Therefore, spraying DA-6 before anthesis or at the seedling stage might affect several different aspects (such as plant height, flowering and seed number per spike) in addition to grain weight. Moreover, the optimal dose of DA-6 at the seedling stage might be lower than that in this study because the biomass per square metre at the seedling stage was far less than that at anthesis. Grain weight is one of the components that determines wheat grain yield. The spike number per hectare and seed number per spike were similar among $\mathrm{C} 0, \mathrm{C} 2, \mathrm{C} 4$, and $\mathrm{C} 6$. Therefore, only seed weight determined grain yield in this study. From

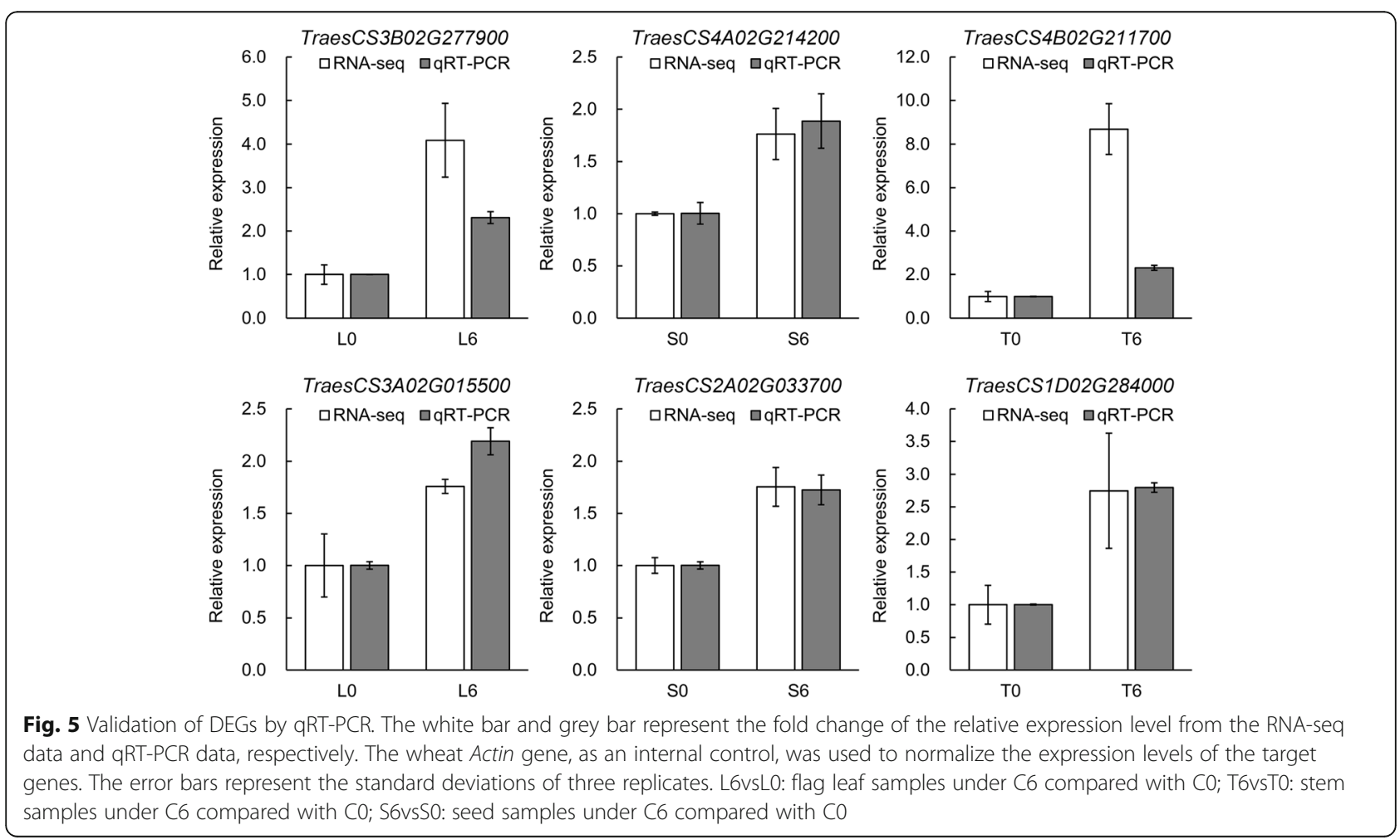


the perspective of grain yield, the optimal stage for spraying DA-6 needs further study.

DA-6 is a plant growth regulator, so an understanding of its receptor and the relationship between DA-6 and plant hormones will help us further understand its action [4]. In this study, the plant hormone signal transduction pathway was the most significantly enriched pathway in the flag leaves. In addition to most of the DEGs involved in ABA signal transduction, one DEG $\left(\right.$ TraesCS6B02G027800, $\log _{2}($ fold change $\left.)=1.6506\right)$ involved in cytokinin signal transduction and one DEG $\left(\right.$ TraesCS2A02G099900, $\log _{2}($ fold change $\left.)=-0.7537\right)$ in volved in ethylene signal transduction were also enriched in the plant hormone signal transduction pathway. Although the plant hormone signal transduction pathway was not significantly enriched in T6vsT0 and S6vsS0, there were more than ten DEGs involved in several plant hormone signal transduction pathways in T6vsT0 and S6vsS0. Plant hormone signal transduction was different among the flag leaves, seeds, and stems in response to DA-6. ABA and cytokinine signal transduction involved DEGs in L6vsL0, T6vsT0, and S6vsS0, and ABA signal transduction revealed more DEGs than did cytokinin signal transduction. Moreover, three DEGs (TraesCS4A02G114400, $\log _{2}$ (fold change $)=-2.6814 ; \quad$ TraesCS4B02G189800, $\quad \log _{2}$ (fold change $)=-2.0476 ; \quad$ TraesCS2D02G087500, $\quad \log _{2}($ fold change $)=-0.84083$ ) in L6vsL0 and two DEGs $\left(\right.$ TraesCS4A02G114400, $\log _{2}($ fold change $)=-1.9287$; TraesCS4D02G191200, $\log _{2}($ fold change $)=-1.6032$ ) in T6vsT0 were implicated in the ABA receptor PYR/PYL family. All five DEGs were markedly downregulated. Therefore, in terms of plant hormones, spraying DA-6 after anthesis in wheat might mainly regulate the ABA signal transduction pathway.

Sucrose is the main product of photosynthesis in wheat, and starch and protein are the major storage nutrients in wheat seed. A previous study showed that sucrose and starch contents in soybean leaves and pods increased from flowering to podding in response to spraying DA-6 at the seedling stage [2]. In this study, spraying DA-6 after anthesis stimulated starch and sucrose metabolism in the flag leaves and seeds. Sucrose synthesis-related DEGs were upregulated in L6vsL0, and starch synthesis-related DEGs were upregulated in S6vsS0. DA-6 increased not only sucrose synthesis in the flag leaves (source organs) but also starch synthesis in the seeds (sink organs), which indicated that DA-6 might enhance the synthesis ability of sucrose and starch. Therefore, the 1000-grain weight under C4 and C6 was significantly higher than that under $\mathrm{C} 0$ during two wheat growing seasons.

Seed protein content affects not only seed processing quality but also seed vigour $[18,19]$. Regardless of the presence or absence of salt stress, DA- 6 treatment significantly increased the concentrations of soluble sugars and soluble protein in Cassia obtusifolia L. [10]. In this study, C4 and C6 had markedly higher seed protein content than did $\mathrm{C} 0$ during two wheat growing seasons. KEGG enrichment analysis showed that protein processing in ER pathway was significantly enriched in both T6vsT0 and S6vsS0. Moreover, DEGs involved in protein processing in ER pathway were mainly involved in ERassociated degradation and the ubiquitin ligase complex in T6vsT0 and S6vsS0. With respect to ER-associated degradation and the ubiquitin ligase complex, the expression of DEGs in T6vsT0 had a higher $\log _{2}$ (fold change) than that in S6vsS0, but S6vsS0 had more DEGs than did T6vsT0. In addition to these DEGs, three other DEGs involved in protein export were also significantly upregulated in S6vsS0. These three DEGs might be associated with protein export from the ER to storage locations. Therefore, the results suggested that DA- 6 regulation of protein processing in ER in the stems and seeds might be related to enhanced seed protein content.

Overall, we propose a model for wheat in response to spraying DA-6 after anthesis (Fig. 6). DA-6 upregulates the expression level of genes involved in sucrose synthesis in the flag leaves, protein processing in ER in the stems, and starch synthesis and protein processing in ER in the seeds. Moreover, sucrose synthesis in the flag leaves might promote starch synthesis in the seeds, and protein processing in ER in the stems might enhance protein processing in ER in the seeds. The increase in starch synthesis and protein processing in ER in the seed enhances grain yield and seed protein content.

\section{Conclusion}

Spraying an appropriate amount of DA-6 at one week after anthesis can increase wheat (Shannong 23) grain yields and seed protein content. Transcriptome analysis revealed that genes involved in sucrose synthesis in the flag leaves, protein processing in ER in the stems, and starch synthesis and protein processing in ER in the seeds were significantly upregulated. Therefore, this study provides new insights into wheat in response to DA-6.

\section{Methods}

\section{Material and experimental design}

Wheat cultivar Shannong 23, which is extensively cultivated in Shandong Province, was used as the experimental material. The seeds were acquired from Shandong Agricultural University. Field experiments were conducted at the experimental station of Shandong Agricultural University (36 $17^{\prime}$ $\mathrm{N}$ and $117^{\circ} 2^{\prime} \mathrm{E}$, Tai'an city, Shandong Province, China) during the wheat growing seasons (October-June) in 20162017 and 2017-2018. Field experiments were laid out in a 


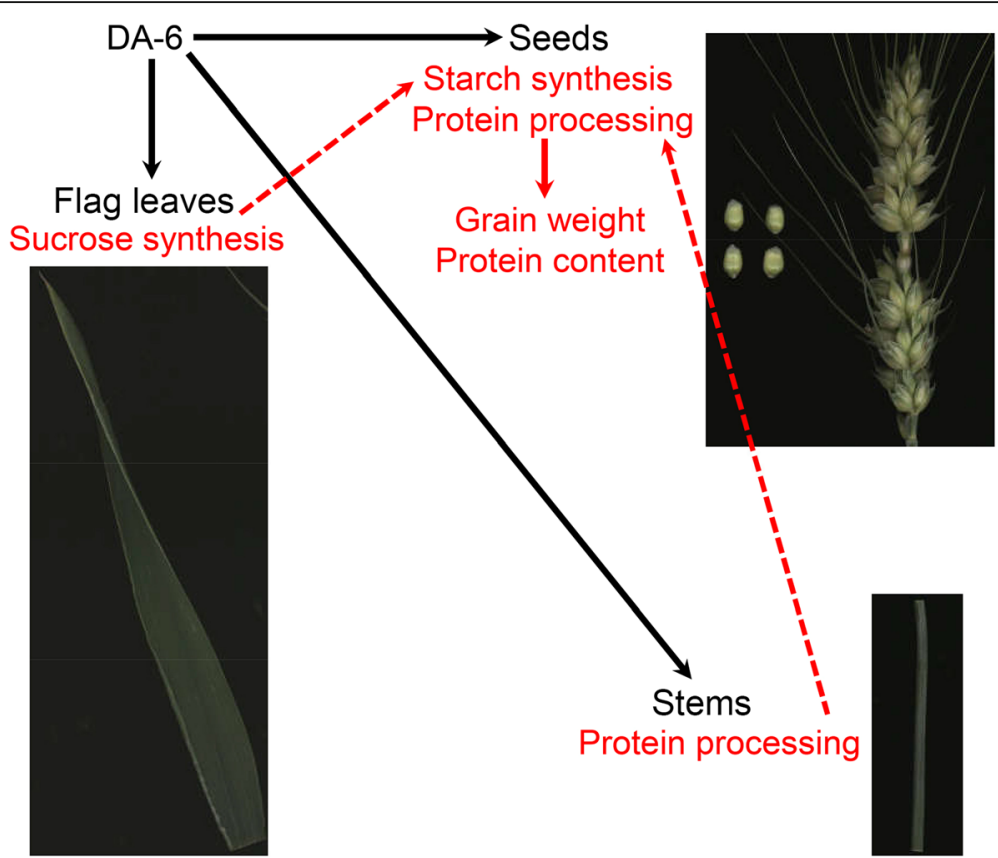

Fig. 6 Possible network by which DA-6 regulates the metabolic pathways in flag leaves, stems, and seeds of wheat. The solid lines indicate definite regulation; the dotted lines indicate conjectural regulation

randomized block design. Each field plot was $2 \mathrm{~m} \times 2 \mathrm{~m}$, and the soil type was a sandy loam. Nitrogen, phosphate, and potash fertilizers were applied as described previously [20]. The monthly rainfall and mean temperature during the two wheat growing seasons are shown in Additional file 3: Figure S3. The irrigation amount under all treatments was equal, and no water stress occurred. DA-6 powder (purity: 98.5\%, Maosheng Chemical Plant, Changzhou, China) was dissolved in distilled water before spraying. We applied four DA6 concentrations: C0 $(0 \mathrm{~g} / \mathrm{L}), \mathrm{C} 2(2 \mathrm{~g} / \mathrm{L}), \mathrm{C} 4(4 \mathrm{~g} / \mathrm{L})$, and C6 $(6 \mathrm{~g} / \mathrm{L})$. Moreover, organic silicone (a surfactant) was added to the DA- 6 solution at a rate of 1 $\mathrm{mL} / \mathrm{L}$. At one week after anthesis (May 2, 2017, and May 3, 2018), the DA-6 solution was sprayed by an electric sprayer with a single nozzle at a rate of 200 $\mathrm{mL} / \mathrm{m}^{2}$ in the afternoon (between $16: 00$ and 18:00, approximately $24{ }^{\circ} \mathrm{C}$ ). The seeds were harvested on June 6, 2017, and June 7, 2018. Diseases, pests, and weeds were well controlled during the two wheat growing seasons.

\section{Grain weight and seed protein content}

A DA7200 device (Perten, Stockholm, Sweden) was used to measure seed moisture contents and seed protein content [19]. One thousand seeds were randomly counted to measure the 1000-grain weight. Each treatment included three replicates. The 1000grain weight was then adjusted to $13 \%$ seed moisture content.
RNA isolation and quality determination

Samples (flag leaves, four seeds located in the middle part of the spike, and approximately $5 \mathrm{~cm}$ long stems below the spikes) of twenty plants were pooled together as one biological sample, and each treatment included three biological replicates. The samples were stored at $-80^{\circ} \mathrm{C}$ after being frozen in liquid nitrogen. RNA extraction, quality determination, and RNA concentration measurements were performed according to a previous study, with some modifications [21]. Total RNA was extracted using TRIzol reagent (Invitrogen, ON, Canada) or a DP441 RNA extraction kit (Tiangen, Beijing, China) according to the manufacturers' protocols. A NanoPhotometer spectrophotometer (IMPLEN, CA, USA) was used to examine RNA purity. Moreover, RNA was monitored on $1 \%$ agarose gels. A Qubit RNA Assay Kit in a Qubit 2.0 Fluorometer (Life Technologies, CA, USA) and an RNA Nano 6000 Assay Kit of the Bioanalyzer 2100 system (Agilent Technologies, CA, USA) were used to measure RNA concentrations and evaluate RNA integrity, respectively. High-quality RNA was used for subsequent RNA-seq library construction.

\section{RNA sequencing and bioinformatics analysis}

RNA-seq library construction, RNA-seq, and bioinformatics analysis were performed as described previously [21]. A NEBNext Ultra ${ }^{\mathrm{Tx}}$ RNA Library Prep Kit for Illumina (NEB, MA, USA) was used for RNA-seq library construction according to the manufacturer's protocol. Index codes were added to the sequences of different samples. Magnetic beads with 
oligo-dT were used to enrich mRNA from approximately $3 \mu \mathrm{g}$ of RNA per sample. Double-stranded cDNAs were synthesized according to the manufacturer's instructions. cDNA fragments that were $150 \sim 200 \mathrm{bp}$ in length were selected by using an AMPure XP system (Beckman Coulter, Beverly, USA). These RNA-seq libraries were sequenced on an Illumina HiSeq platform for generating $150 \mathrm{bp}$ paired-end reads. Reads containing adapters, reads containing poly- $\mathrm{N}$, and low-quality reads were removed from the raw reads. Moreover, the Q20, Q30 and GC contents of the clean data were also calculated. The clean reads were subsequently mapped to the wheat genome sequence (ftp://ftp.ensemblgenomes. org/pub/plants/release-41/fasta/triticum_aestivum/dna/) by using TopHat v2.0.12 [22]. The read numbers of each gene were counted by using HTSeq v0.6.1. The DESeq $R$ package (1.18.0) was used for performing a differential expression analysis of the two groups [23, 24]. Benjamini and Hochberg's approach was then used to adjust the resulting $p$-values for controlling the false discovery rate. Genes with an adjusted $p$-value $<0.05$ were considered differentially expressed genes (DEGs). GO and KEGG enrichment analyses were carried out based on the DEGs. The GO enrichment analysis of DEGs was performed by using the GOseq $\mathrm{R}$ package, and GO terms with corrected $p$-values $<0.05$ were assigned as significantly enriched GO terms [25]. The KEGG enrichment analysis of the DEGs was performed by using KOBAS software [26]. Similarly, the KEGG pathways with corrected $p$-values $<0.05$ were assigned as significantly enriched pathways.

\section{qRT-PCR}

Validation of the gene expression levels revealed by RNA-seq was carried out by qRT-PCR. An ABI StepOne Plus Real-Time PCR System (Applied Biosystems, CA, USA) was used to perform all qRT-PCR analyses. Genespecific primers for qRT-PCR were synthesized by Sangon Biotech (Shanghai, China) and are listed in Additional file 8: Table S5. A PrimeScript RT reagent kit (Takara, Dalian, China) was used to synthesize cDNA from total RNA. The wheat Actin gene, as an internal control, was used to normalize the expression levels of the target genes [27]. Each qRT-PCR experiment was repeated three times, and the mean expression level and standard deviation were calculated using the $2^{-\triangle \Delta \mathrm{Ct}}$ method [28].

\section{Statistical analysis}

One-way analysis of variance and Duncan's multiple tests were performed by using SPSS 19.0 software (IBM, New York, USA).

\section{Additional files}

Additional file 1: Figure S1 Top 30 significantly enriched $\mathrm{GO}$ terms in L6vsLO. (DOCX $1233 \mathrm{~kb}$ )

Additional file 2: Figure S2 Significantly enriched GO terms in T6vsTO. (DOCX $1122 \mathrm{~kb}$ )

Additional file 3: Figure S3 Meteorological data recorded during the period of wheat growth (October to June). (DOCX $708 \mathrm{~kb}$ )

Additional file 4: Table S1 Summary of RNA-seq data. (DOCX $15 \mathrm{~kb}$ )

Additional file 5: Table S2 List of selected genes for KEGG pathways in L6vsLO. (DOCX $14 \mathrm{~kb}$ )

Additional file 6: Table S3 List of selected genes for KEGG pathways in T6vsT0. (DOCX $15 \mathrm{~kb}$ )

Additional file 7: Table S4 List of selected genes for KEGG pathways in S6vsSO. (DOCX $16 \mathrm{~kb}$ )

Additional file 8: Table S5 Primers used for qRT-PCR. (DOCX 14 kb)

\section{Abbreviations}

DA-6: Diethyl aminoethyl hexanoate; DEG: differentially expressed gene;

ER: endoplasmic reticulum; GO: Gene Ontology; KEGG: Kyoto Encyclopedia of Genes and Genomes; L6vsL0: flag leaf samples under C6 compared with C0; S6vsSO: seed samples under C6 compared with C0; T6vsT0: stem samples under C6 compared with CO

\section{Acknowledgements}

We thank the funding support.

\section{Authors' contributions}

DW, YL and CZ conceived the study. DW, YL and LH performed the experiments and analysed the results. DW and CZ wrote the manuscript, with feedback from all authors. All authors have read and approved the manuscript.

\section{Funding}

This work was supported by the National Key Research and Development Plan of China (number 2018YFD0100900) and the Special Fund of Agricultural Significant Application Technique Innovation of Shandong Province. The funding bodies were not involved in the design of the study, collection, analysis, interpretation of data, and in writing the manuscript.

\section{Availability of data and materials}

The datasets generated and/or analysed in this study are included in the article and additional files. The RNA-seq data have been deposited in the NCBI Sequence Read Archive (SRA, http://www.ncbi.nlm.nih.gov/sra) under accession number PRJNA544398.

Ethics approval and consent to participate

Not applicable.

Consent for publication

Not applicable.

\section{Competing interests}

The authors declare that they have no competing interests.

Received: 19 June 2019 Accepted: 5 July 2019

Published online: 19 July 2019

\section{References}

1. Jiang Y, Jiang Y, He S, Zhang H, Pan C. Dissipation of diethyl aminoethyl hexanoate (DA-6) residues in pakchoi, cotton crops and soil. B Environ Contam Tox. 2012;88(4):533-7.

2. Liu C, Feng N, Zheng D, Cui H, Sun F, Gong X. Uniconazole and diethyl aminoethyl hexanoate increase soybean pod setting and yield by regulating sucrose and starch content. J Sci Food Agr. 2019;99(2):748-58.

3. Qi RJ, Gu WR, Zhang J, Ling H, Zhang MC, Duan LS, Li ZH. Exogenous diethyl aminoethyl hexanoateenhanced growth of corn and soybean 
seedlings through altered photosynthesis and phytohormone. Aust J Crop Sci. 2013;7(13):2021-8.

4. Zhou W, Chen F, Zhao S, Yang C, Meng Y, Shuai H, Luo X, Dai Y, Yin H, Du $J$, et al. DA-6 promotes germination and seedling establishment from aged soybean seeds by mediating fatty acid metabolism and glycometabolism. J Exp Bot. 2019;70(1):101-14.

5. Jiang L, Pei H, Hu W, Han F, Zhang L, Hou Q. Effect of diethyl aminoethyl hexanoate on the accumulation of high-value biocompounds produced by two novel isolated microalgae. Bioresour Technol. 2015;197:178-84.

6. Ding W, Peng J, Zhao Y, Zhao P, Xu J, Li T, Yu X. A strategy for boosting astaxanthin accumulation in green microalga Haematococcus pluvialis by using combined diethyl aminoethyl hexanoate and high light. J Appl Phycol. 2019;31(1):171-81.

7. Fu XJ, Maimaiti AS, Mou HM, Yang Q, Liu GJ. Hexanoic acid 2(diethylamino)ethyl ester enhances chilling tolerance in strawberry seedlings by impact on photosynthesis and antioxidants. Biol Plantarum 2011:55(4):793-6.

8. He S, Wu Q, He Z. Synergetic effects of DA-6/GA3 with EDTA on plant growth, extraction and detoxification of cd by Lolium perenne. Chemosphere. 2014;117:132-8.

9. Li Z, Zhang $\mathrm{R}$, Zhang $\mathrm{H}$. Effects of plant growth regulators (DA-6 and 6-BA) and EDDS chelator on phytoextraction and detoxification of cadmium by Amaranthus hybridus Linn. Int J Phytoremediat. 2018;20(11):1121-8.

10. Zhang C, He P, Li Y, Li Y, Yao H, Duan J, Hu S, Zhou H, Li S. Exogenous diethyl aminoethyl hexanoate, a plant growth regulator, highly improved the salinity tolerance of important medicinal plant Cassia obtusifolia L. J Plant Growth Regul. 2016;35(2):330-44.

11. He S, Wu Q, He Z. Growth-promoting hormone DA-6 assists phytoextraction and detoxification of cd by ryegrass. Int J Phytoremediat. 2015;17(6):597-603.

12. Xu C, Gao Y, Tian B, Ren J, Meng Q, Wang P. Effects of EDAH, a novel plant growth regulator, on mechanical strength, stalk vascular bundles and grain yield of summer maize at high densities. Field Crop Res. 2017;200:71-9.

13. Salama E, Jeon B, Chang SW, Lee S, Roh H, Yang I, Kurade MB, El-Dalatony MM, Kim D, Kim K, et al. Interactive effect of indole-3-acetic acid and diethyl aminoethyl hexanoate on the growth and fatty acid content of some microalgae for biodiesel production. J Clean Prod. 2017:168:1017-24.

14. Zhang H, Xie L, Xu P, Jiang S. Dissipation of the plant growth regulator hexanoic acid 2-(diethylamino) ethyl ester in pakchoi and soil. Int J Environ An Ch. 2008;88(8):561-9.

15. Li N, Li Y. Signaling pathways of seed size control in plants. Curr Opin Plant Biol. 2016;33:23-32.

16. Zhang Y, Dai X, Jia D, Li H, Wang Y, Li C, Xu H, He M. Effects of plant density on grain yield, protein size distribution, and breadmaking quality of winter wheat grown under two nitrogen fertilisation rates. Eur J Agron. 2016;73:1-10.

17. Dai Z, Plessis A, Vincent J, Duchateau N, Besson A, Dardevet M, Prodhomme D, Gibon Y, Hilbert G, Pailloux M, et al. Transcriptional and metabolic alternations rebalance wheat grain storage protein accumulation under variable nitrogen and sulfur supply. Plant J. 2015;83(2):326-43.

18. Li D, Jin H, Zhang K, Wang Z, Wang F, Zhao Y, Huo N, Liu X, Gu YQ, Wang $D$, et al. Analysis of the Gli-D2 locus identifies a genetic target for simultaneously improving the breadmaking and health-related traits of common wheat. Plant J. 2018:95(3):414-26.

19. Wen D, Hou H, Meng A, Meng J, Xie L, Zhang C. Rapid evaluation of seed vigor by the absolute content of protein in seed within the same crop. Sci Rep-Uk. 2018;8:5569.

20. Zheng M, Chen J, Shi Y, Li Y, Yin Y, Yang D, Luo Y, Pang D, Xu X, Li W, et al. Manipulation of lignin metabolism by plant densities and its relationship with lodging resistance in wheat. Sci Rep-Uk. 2017;7:41805.

21. Wen D, Xu H, Xie L, He M, Hou H, Zhang C. A loose endosperm structure of wheat seed produced under low nitrogen level promotes early germination by accelerating water uptake. Sci Rep-Uk. 2017;7:3116.

22. Trapnell C, Pachter L, Salzberg SL. TopHat: discovering splice junctions with RNA-Seq. Bioinformatics. 2009;25(9):1105-11.

23. Anders S, Huber W. Differential expression analysis for sequence count data. Genome Biol. 2010;11:R106.

24. Wang L, Feng Z, Wang X, Wang X, Zhang X. DEGseq: an R package for identifying differentially expressed genes from RNA-seq data. Bioinformatics, 2010;26(1):136-8

25. Young MD, Wakefield MJ, Smyth GK, Oshlack A. Gene ontology analysis for RNA-seq: accounting for selection bias. Genome Biol. 2010;11(2):R14.
26. Mao X, Cai T, Olyarchuk JG, Wei L. Automated genome annotation and pathway identification using the KEGG Orthology $(\mathrm{KO})$ as a controlled vocabulary. Bioinformatics. 2005;21(19):3787-93.

27. Wang C, Wen D, Sun A, Han X, Zhang J, Wang Z, Yin Y. Differential activity and expression of antioxidant enzymes and alteration in osmolyte accumulation under high temperature stress in wheat seedlings. I Cereal Sci. 2014;60(3):653-9.

28. Livak KJ, Schmittgen TD. Analysis of relative gene expression data using real-time quantitative PCR and the $2^{-\Delta \Delta C T}$ method. Methods. 2001;25:402-8.

\section{Publisher's Note}

Springer Nature remains neutral with regard to jurisdictional claims in published maps and institutional affiliations.
Ready to submit your research? Choose BMC and benefit from:

- fast, convenient online submission

- thorough peer review by experienced researchers in your field

- rapid publication on acceptance

- support for research data, including large and complex data types

- gold Open Access which fosters wider collaboration and increased citations

- maximum visibility for your research: over $100 \mathrm{M}$ website views per year

At BMC, research is always in progress.

Learn more biomedcentral.com/submissions 